\title{
Strain-rate dependent mechanism of cooperative dislocation generation: application to the brittle-ductile transition
}

\author{
M. Khantha*, V. Vitek, D.P. Pope \\ Department of Materials Science and Engineering, University of Pennsylvania, Philadelphia, PA 19104-6272, USA
}

\begin{abstract}
A strain-rate dependent mechanism of cooperative dislocation generation in loaded solids above a critical temperature is described. The massive dislocation activity, which commences near the crack tip at the brittle-to-ductile transition temperature is modeled in terms of this mechanism. The strain-rate dependence of the critical temperature arises from the glide of both pre-existing dislocations and dislocations which are 'thermally nucleated' below the critical temperature by the cooperative process. Depending on their relative contributions, the apparent activation energy associated with the brittle-to-ductile transition temperature is either equal to or larger than the activation energy for dislocation motion. We compare the predictions of the model with observations in TiAl. (C) 2001 Elsevier Science B.V. All rights reserved.
\end{abstract}

Keywords: Cooperative dislocation generation; Brittle-ductile transition; Strain-rate effects

\section{Introduction}

The brittle-to-ductile transition (BDT) [1] exhibited by most materials is associated with two distinct features, (i) the transition is marked by a dramatic increase in the dislocation activity at the crack tip concomitant with a rapid increase of the fracture toughness [2]; and (ii) an apparent activation energy can be associated with the strain-rate dependence of the brittle-to-ductile transition temperature (BDTT). This is often, but not always, the same as the activation energy for dislocation motion. For example, in silicon and other semiconductors $[2,3]$, the activation energy associated with the BDTT is almost exactly equal to the activation energy for dislocation motion. However, in TiAl single crystals [4], the activation energy associated with the BDTT is $1.4 \mathrm{eV}$ when the slip is dominated by ordinary dislocations and $4.9 \mathrm{eV}$ when it is dominated by superdislocations. Despite this difference in the activation energies, the BDTT, while varying with the strain rate, remains in the same temperature range, viz. between 516-750 and $635-685^{\circ} \mathrm{C}$ for ordinary and superlattice dislocations, respectively [4].

\footnotetext{
* Corresponding author. Tel.: + 1-215-8982913; fax: + 1-2155732128.

E-mail address: khantha@1rsm.upenn.edu (M. Khantha).
}

We have recently proposed a cooperative dislocation generation process in loaded crystals that can lead above a critical temperature to the formation and expansion of many dislocation loops without any energy barrier [5-8]. In the present paper, we advance this development to a new level in which dislocation generation occurs in a strain-rate dependent manner. This comprehensive model is then used to explain the BDT and predict the BDTT. The model also shows why in certain situations, the apparent activation energy associated with the BDTT can be substantially higher than the activation energy for dislocation motion.

\section{Synopsis of the cooperative dislocation generation model}

The density of homogeneously nucleated dislocation loops of radius $r$ is proportional to the Boltzmann factor, $\exp \left[-H(r) / k_{\mathrm{B}} T\right]$, where $H(r)$ is the formation enthalpy of the loop, $k_{\mathrm{B}}$, the Boltzmann constant and $T$, the temperature. For an isolated shear loop, when the material is loaded by a shear stress $\sigma$ in the direction of the Burgers vector,

$H(r)=K_{0}\left[r \ln \left(\frac{r}{r_{0}}\right)+c r\right]-\sigma b \pi r^{2}$ 
where $K_{0}=\left[\mu_{0}\left(2-v_{0}\right) b^{2} / 4\left(1-v_{0}\right)\right], b$ is the magnitude of the Burgers vector, $\mu_{0}$ the elastic shear modulus in the slip plane, $v_{0}$ the Poisson ratio, $K_{0} c$ the core energy of the dislocation and $r_{0}$ the cut-off radius. The second term in Eq. (1) is the work done by the external stress and thus the density of loops at a given temperature is higher in the loaded solid. Dislocation loops of atomic sizes have finite formation energies typically in the range $0.5-3.0 \mathrm{eV}$ and can thus form by spontaneous fluctuations at finite temperatures. However, these loops are sub-critical in size and usually shrink and disappear. When the applied stress is much smaller than the ideal shear strength, the activation barrier for the unstable expansion of individual loops is large, of the order of $10 \mathrm{eV}$. Hence, the homogeneous nucleation of individual dislocations is highly improbable as always assumed.

The cooperative dislocation generation represents the simultaneous nucleation and subsequent evolution of many sub-critical dislocation loops that form at finite temperatures in a loaded solid. This process, which occurs owing to the combined effect of dislocation interactions and entropy in both the nucleation and evolution stages, is manifestly different from the nucleation and unstable expansion of an individual dislocation loop. The analytical treatment of dislocation interactions is a formidable but tractable task [9]. There is, however, a simpler approach based on the mean field theory $[10,11]$ where the effect of dislocation interactions is probed indirectly in two steps. First, a crystal with 'effective' properties is defined. These properties reflect the presence and interactions of many subcritical dislocation loops. In the second step, the behavior of test dislocation loops in this 'effective' medium is investigated. Using the renormalization group technique of statistical mechanics, it can be shown that the $N$-body Hamiltonian describing the self and interaction energies of $N$ dislocation loops is equivalent to the Hamiltonian of a test loop in a medium with appropriate effective properties $[9,12]$.

The effective properties are identified in the following way [6]. When a dislocation loop is formed in a medium containing other loops, a rearrangement of the existing loops takes place by contraction or expansion under the influence of the stress field of the newly formed loop. Under applied loads there is a net plastic strain associated with this rearrangement. This additional strain is responsible for a decrease of the 'effective moduli' that relate stresses and total strains in the medium. The self energy of the test loop in the effective medium is proportional to the effective moduli just as the energy of an isolated dislocation loop is proportional to the elastic moduli. Thus, the formation enthalpy of a test loop in the effective medium can be written as in Eq. (1) but with $K_{0}$ replaced by $K_{\text {eff }}=K_{0} / \varepsilon$, where $\varepsilon$ is akin to a 'dielectric function' and is the energy coefficient written in terms of the effective moduli. $\varepsilon$ describes the decrease of the self energy of the test loop due to the plastic strain associated with other loops.

At low temperatures, the difference between the effective and elastic moduli is very small and $\varepsilon$ is nearly equal to unity, which is its minimum value. As the temperature increases, the probability of formation of dislocation loops increases in the loaded crystal, which in turn, increases the strain of plastic type in the medium. Consequently, the effective moduli decrease and $\varepsilon$ increases. As a result, the formation enthalpy of the test loop in the effective medium is smaller than that of an isolated loop of the same size. In the $N$-body description, this corresponds to the reduction of the formation energy of a dislocation loop present amidst other loops owing to their mutual interactions. This, in turn, promotes the formation of more dislocation loops in the crystal, which further increases the plastic strain. A positive or 'cooperative' feedback is set up between the formation of additional sub-critical dislocation loops and the continued reduction of the effective moduli. Concomitant with the reduction of the formation energy, the activation barrier and the critical radius for expansion of the test loop decrease progressively as the temperature increases (in contrast, the activation barrier for the expansion of a single dislocation loop does not vary with temperature.) At the same time, the entropy of the dislocation loops increases as their number increases with temperature. Ultimately, at a critical temperature, $T_{\mathrm{c}}$, the free energy of the test loop vanishes. The unstable expansion and glide of the test loop in the effective medium above $T_{\mathrm{c}}$ corresponds to the spontaneous nucleation and glide of many dislocation loops in the stressed crystal. This massive dislocation activity makes the effective moduli approach zero or $\varepsilon$ diverge to infinity above $T_{\mathrm{c}}$.

This mechanism of cooperative dislocation generation can operate in all crystalline materials, both in the presence and absence of applied loads. In a material that is not loaded, this process is the well-known Kosterlitz-Thouless $(\mathrm{K}-\mathrm{T})$ defect-mediated phase transition [10,12,13], which occurs just below the melting temperature. This transition was first recognized in two dimensions but the mechanism was later generalized to three dimensions [14]. The principal difference between the $\mathrm{K}-\mathrm{T}$ type models and our approach [5$8,15]$ is the presence of external loads which enables the cooperative instability to take place well below the melting temperature in both two and three dimensions.

\subsection{Strain-rate effects}

In our previous studies, we have developed a static description of the cooperative instability in dislocationfree two [6] and three-dimensional (3-D) crystals [7,8]. 
The application of this mechanism to the BDT [15] showed that the model can explain the sudden and massive onset of dislocation activity at the BDTT. The estimated critical temperature for the cooperative instability was in the same range as the BDTT in a variety of brittle materials. However, the model could not predict the strain-rate dependence of the BDTT since dynamical effects were not included. In this paper, we incorporate the effect of dislocation dynamics and investigate how this affects the onset of the cooperative instability. Two types of dislocation activity influence the dynamics (i) the glide of pre-existing dislocations below $T_{\mathrm{c}}$ affects the onset of the cooperative instability by contributing to the plastic strain similarly as do the sub-critical dislocation loops; (ii) glissile dislocations can be generated by thermal activation at temperatures below $T_{\mathrm{c}}$ since the activation barrier is reduced owing to the cooperative interactions. This barrier falls below $2 \mathrm{eV}$ at temperatures $100-200 \mathrm{~K}$ below $T_{\mathrm{c}}$. The glide of such 'thermally nucleated' dislocations also contributes to the plastic strain.

In order to consider the effect of dislocation dynamics, it is necessary to specify the time period over which dislocation glide occurs and contributes to the plastic strain. In applications to the BDT, an appropriate choice is the time that elapses before the stress intensity reaches the critical value for fracture at a given loading rate. Let the contributions to $\varepsilon$ from the glide of pre-existing dislocations of density $n_{0}$ and thermally nucleated 'free' dislocations of density $n_{f}$ during a specified time interval be denoted by $\chi_{0}$ and $\chi_{f}$, respectively. The 'dielectric function' $\varepsilon$ can then be decomposed as

$\varepsilon=1+4 \pi \chi_{0}+4 \pi \chi_{b}+4 \pi \chi_{f}$

$\chi_{b}$ represents the contribution from sub-critical dislocation loops formed by thermal fluctuations. The static model [6,7] did not include the contributions $\chi_{0}$ and $\chi_{f}$. In this limit, $\chi_{b}$ depends only on the number of sub-critical dislocation loops present which in turn is affected by the value of $\varepsilon$. Thus, the self-consistent determination of $\varepsilon$ is the crucial step in the analysis of the cooperative instability. This step gains further importance in the dynamical model because $n_{f}$ and its associated contribution $\chi_{f}$ depend on the reduced activation barrier which in turn is determined by $\varepsilon$. In addition, the number of sub-critical loops and hence $\chi_{b}$ at any temperature depend on both $n_{0}$ and $n_{f}$.

For a given set of material parameters and fixed external loads, the cooperative instability occurs when $\varepsilon$ attains a critical value, $\varepsilon_{\mathrm{c}}$. Thus, from Eq. (2), we see that the temperature at which the cooperative instability occurs is determined by how much $\chi_{0}$ and $\chi_{f}$ contribute towards $\varepsilon_{\mathrm{c}}$. Both these contributions are strain-rate or time-dependent and, thus, the BDTT predicted by this model is expected to be strain-rate dependent. Since $\chi_{0}$ and $\chi_{f}$ determine the critical temperature, the activation energy associated with the strain-rate dependence of the BDTT depends on the activation energy, $U_{m}$ for dislocation motion and the apparent formation energy, $U_{n}$, associated with the thermally nucleated dislocations of density, $n_{f}$, at the BDTT. However, $U_{n}$ is not a constant but is temperature-dependent and its value is determined by the effectiveness of dislocation interactions in reducing the energy barrier of test loops. Thus, $U_{n}$ depends on $\varepsilon$ and we seek a self-consistent solution to this complex problem.

We begin with a summary of equations that describe the static 3-D model [7]. The number density, $n(r)$, of test dislocation loops of radius $r$ at temperature $T$ is proportional to the Boltzmann factor, $\exp \left[-H_{\text {eff }}(r) /\right.$ $k_{\mathrm{B}} T$ ] where $H_{\text {eff }}(r)$ is the enthalpy of a test loop of radius $r$ given by

$H_{\text {eff }}(r)=\int_{r_{0}}^{r}\left(\frac{K_{0}}{r_{0} \varepsilon\left(r^{\prime}\right)}\right)\left\{\ln \left(\frac{r^{\prime}}{r_{0}}\right)+c+1\right\} \mathrm{d} r^{\prime}-\sigma b \pi r^{2}$

The determination of $\varepsilon$ is made easier by identifying the following variables:

$$
\begin{gathered}
\ell=\ln \left(\frac{r}{r_{0}}\right) ; \quad y(\ell)=\left(\frac{r}{r_{0}}\right)^{6} \exp \left(\frac{-H_{\mathrm{eff}}(r)}{k_{\mathrm{B}} T}\right) ; \\
h(\ell)=\frac{K_{0} r}{k_{\mathrm{B}} T \varepsilon(r)}
\end{gathered}
$$

The logarithmic length scale defined by $\ell$ follows naturally for dislocation type interactions. The function $y(\ell)$ is proportional to the number density of test dislocation loops and $h(\ell)$ is a measure of the effective interaction coefficient $K_{\text {eff }}$. In terms of these variables, the self-consistent solution to $\varepsilon$ and the determination of the critical temperature in the static limit can be re-cast as the solution to a system of coupled differential equations [7],

$\frac{\mathrm{d} h}{\mathrm{~d} \ell}=h-\frac{h^{2} y \pi^{3}}{96} ; \quad \frac{\mathrm{d} y}{\mathrm{~d} \ell}=\left[6-\frac{h(\ell+c+1)}{4}+2 \sigma b \pi r^{2} \beta\right] y$

We now consider the time-dependent contributions to $\varepsilon$ arising from the glide of pre-existing and thermally nucleated dislocations. The plastic strain due to the glide of dislocations of density $n_{0}$ in a specified time interval is equal to the product of $n_{0}$, the Burgers vector $b$ and the distance traveled. It was shown in an earlier work [16] that the dynamics of interacting dislocations near a loaded crack is of the 'similarity type' when the velocity of dislocations $(v)$ has the form $v=A\left(\sigma / \sigma_{0}\right)^{m}$ $\exp \left(-U_{m} / k_{\mathrm{B}} T\right)$ where $A$ and $\sigma_{0}$ are constants and $U_{m}$ is the activation energy associated with the dislocation motion. Accordingly, if $N$ dislocations are at positions $X_{i}^{0}(i=1,2, \ldots, N)$ at time $t=0$, then their positions at time $t$ when the crack is subject to a constant loading rate $[16,17]$ are given by $X_{i}(t) \propto X_{i}^{0} g(t)$ where $g(t)=$ 
$\left(\exp \left[-U_{m} / k_{\mathrm{B}} T\right] \Lambda t\right)^{2(m+1) /(m+2)}$ and $\Lambda$ is a constant. Using this solution, the contributions to $\varepsilon$ from $\chi_{f}$ and $\chi_{0}$ can be estimated. To make the model analytically tractable, we make the following assumptions (i) the plastic strain arising from sub-critical loops of size less than or equal to $r$ is assumed to be time independent and the same for all loading rates. While this is clearly an approximation, it is reasonable in the present context because this term makes the smallest contribution to $\varepsilon$ compared with $\chi_{f}$ and $\chi_{0}$; (ii) in accordance with the previous assumption, the minimum size of the dislocation loop which expands and glides macroscopically is assumed to be $r$. In other words, $X_{i}^{0}$ is set equal to $r$ for both pre-existing and thermally nucleated dislocations. It is then straightforward to estimate the contributions $\chi_{f}$ and $\chi_{0}$ using the same definitions as in the static model [6]. The result is

$$
\varepsilon(r)=1+4 \pi \chi_{b}(r)+\left(\mu_{0} \frac{\left(2-v_{0}\right)}{\left(1-v_{0}\right) \sigma}\right)\left[n_{f}+n_{0}\right] \operatorname{bMrg}(t)
$$

where $t$ is the minimum time available at a given loading rate before fracture occurs and $M$ is a constant.

The density of thermally nucleated dislocations, $n_{f}$, is calculated from a Fokker-Planck equation which describes the escape over the energy barrier for the unstable expansion of a test loop in the effective medium. This procedure has been outlined in the so-called AHNS model [18] which was first proposed to describe the dynamics of the $\mathrm{K}-\mathrm{T}$ transition in an applied field. If $r_{\mathrm{c}}$ denotes the critical radius above which the test loop can expand in an unstable manner at a given temperature, then $r_{\mathrm{c}}$ and $n_{f}$ are given by

$r_{\mathrm{c}}=\left(\frac{K_{0}}{8 r_{0} \sigma b \pi \varepsilon\left(r_{\mathrm{c}}\right)}\right)\left(\ln \frac{r_{\mathrm{c}}}{r_{0}}+c+1\right) ; \quad n_{f}=\frac{\sqrt{y\left(r_{\mathrm{c}}\right)}}{r_{\mathrm{c}}^{2}}$

Having identified all the contributions to $\varepsilon$ in Eq. (2), the next step is to determine the new dynamically coupled differential equations for the variables defined by Eq. (4). Owing to the contributions from $\chi_{f}$ and $\chi_{0}$, the equation for $\mathrm{d} h / \mathrm{d} \ell$ differs from the static case (see Eq. (5)) while the equation for $\mathrm{d} y / \mathrm{d} \ell$ remains the same. There is a complex interdependence of $\varepsilon$ (see Eqs. (2)-(7)) which makes the self-consistent determination of $\varepsilon$ very difficult. We break this process into the

Table 1

The strain-rate dependence of the BDTT in TiAl

\begin{tabular}{llllll}
\hline $\begin{array}{l}\text { Strain rate } \\
\left(\mathrm{s}^{-1}\right)\end{array}$ & \multicolumn{2}{l}{$n_{i}=1.25 \times 10^{8} \mathrm{~m}^{-2}$} & & \multicolumn{2}{l}{$n_{i}=1.25 \times 10^{10} \mathrm{~m}^{-2}$} \\
\cline { 2 - 3 } & BDTT $(\mathrm{K})$ & $n_{f}\left(\mathrm{~m}^{-2}\right)$ & & BDTT $(\mathrm{K})$ & $n_{f}\left(\mathrm{~m}^{-2}\right)$ \\
\hline $1.2 \times 10^{-5}$ & 763 & $6.0 \times 10^{9}$ & 672 & $1.1 \times 10^{11}$ \\
$1.2 \times 10^{-4}$ & 800 & $7.4 \times 10^{10}$ & 746 & $2.5 \times 10^{11}$ \\
$1.2 \times 10^{-3}$ & 820 & $9.0 \times 10^{11}$ & 807 & $1.2 \times 10^{12}$ \\
$1.2 \times 10^{-2}$ & 840 & $1.2 \times 10^{13}$ & 839 & $1.3 \times 10^{13}$ \\
\hline
\end{tabular}

following two parts in order to make the model tractable. First, we determine $r_{\mathrm{c}}$ and $n_{f}$ using only the contributions $\chi_{0}$ and $\chi_{b}$ and evaluate $\varepsilon$ self-consistently. In the next step, we use the values of $n_{f}$ and $\chi_{f}$ in addition to the contributions from $\chi_{b}$ and $\chi_{0}$ and obtain a self-consistent solution to $\varepsilon$.

\section{Results and discussion}

We have solved the dynamical model using the approach summarized above with parameters appropriate for TiAl. We consider only ordinary dislocations and accordingly set $b=2.83 \AA ; \mu_{0}=70 \mathrm{GPa}$ and $v_{0}=0.23$ represent average values for polycrystalline $\mathrm{TiAl}$; the elastic cut-off radius is taken to be the same as the Burgers vector $\left(r_{0}=2.83 \AA\right.$ ) and $c=0.25$ such that the formation enthalpy of an isolated loop of radius $3.4 \AA$ is approximately $1 \mathrm{eV}$. We assume further that the material is loaded by a constant stress, $\sigma=3 \mathrm{GPa}$. Such large stress levels are expected in the vicinity of a crack. The activation barrier and the critical radius, $r_{\mathrm{c}}$, for unstable expansion of an isolated loop are $22 \mathrm{eV}$ and 20 $\AA$, respectively, at $300 \mathrm{~K}$. At the same temperature, the corresponding values for a test loop in the effective medium are only slightly lower than those for an isolated loop. However, as the temperature increases, the activation energy and $r_{\mathrm{c}}$ decrease dramatically for the test loop and at $1200 \mathrm{~K}$, they are $0.15 \mathrm{eV}$ and $3.1 \AA$, respectively. We now describe how the glide of pre-existing and thermally nucleated dislocations influences the strain-rate dependence and the apparent activation energy associated with the BDTT.

The BDT measurements in TiAl [4] show that the critical stress intensity for fracture is approximately 2.5 $\mathrm{MPa} \sqrt{ } \mathrm{m}$ for strain rates between $10^{-5}-10^{-3} \mathrm{~s}^{-1}$. In the above experiment, a strain rate of $1.2 \times 10^{-5} \mathrm{~s}^{-1}$ corresponds to a rate of stress intensity $2.5 \times 10^{3} \mathrm{~Pa}$ $\sqrt{\mathrm{ms}^{-1}}{ }^{-}$. Thus, the approximate time scale over which glide of dislocations takes place before fracture occurs at this strain rate is $t=10^{3} \mathrm{~s}$. The time scale at other strain rates can be found by suitable increments or decrements from this baseline value. Since the dislocation mobility is not known in TiAl, we set the values for various parameters in such a manner as to give reasonable values for the mobility. Accordingly, $M=$ $1 \times 10^{11}, U_{m}=1 \mathrm{eV}$, the velocity-stress exponent $m=1$ and the constant $\Lambda=10$. We consider two values of $n_{0}=1.25 \times 10^{8}$ and $1.25 \times 10^{10} \mathrm{~m}^{-2}$, respectively. The calculated strain-rate dependence of the BDTT along with the values of $n_{f}$ at this temperature are given in Table 1 for the two cases.

The apparent activation energy, $U_{a p p}$, associated with the strain-rate dependence of the BDTT can be obtained by plotting the logarithm of the strain rate versus 1/BDTT. $U_{a p p}=4.9 \mathrm{eV}$ for $n_{f}=1.25 \times 10^{8} \mathrm{~m}^{-2}$ 
and $U_{a p p}=1.57 \mathrm{eV}$ for $n_{i}=1.25 \times 10^{10} \mathrm{~m}^{-2}$. These very different values of $U_{a p p}$ stem from the apparent formation energies associated with the density of thermally nucleated dislocations, $n_{f}$, in the two cases. As mentioned earlier, the thermally nucleated dislocations are formed because of the reduction of the activation energy due to dislocation interactions and this process depends both on temperature and $\varepsilon$. The results of the dynamical model show that the variation of $n_{f}$ at the BDTT for different strain rates has the form $n_{f} \propto \exp$ ( $-U_{n} / k_{\mathrm{B}} T_{\mathrm{c}}$ ). We identify $U_{n}$ as an apparent formation energy since the above description is phenomenological and not indicative of the process by which they form [18]. Using the data in Table 1 , we find that $U_{n}=5.3 \mathrm{eV}$ for $n_{i}=1.25 \times 10^{8} \mathrm{~m}^{-2}$ and $U_{n}=0.79 \mathrm{eV}$ for $n_{i}=$ $1.25 \times 10^{10} \mathrm{~m}^{-2}$. The contribution $\chi_{0}$ is much larger when $n_{0}$ is larger and this causes a dramatic reduction in $U_{n}$ which, in turn, helps to lower the BDTT by nearly $100 \mathrm{~K}$ at low strain rates. Using the analytical form for the similarity dynamics, it can be shown that $U_{\text {app }}=U_{m}+(m+2) U_{n} / 2(m+1)$ which is in excellent agreement with the results obtained above. Thus, $U_{a p p}$ can either be equal to $U_{m}$ when $U_{n}$ is nearly zero and/or it can be substantially higher than $U_{m}$ depending on the value of $U_{n}$ and the exponent $m$.

The BDTT measurements in TiAl [4] reveal very different values for $U_{a p p}$ for ordinary and superlattice dislocations (1.4 and $4.9 \mathrm{eV}$, respectively) even though the BDTT lies in the same range for both cases. The cooperative dislocation generation model shows that $U_{\text {app }}$ represents a composite value determined by many inter-related thermally activated processes. Thus, $U_{a p p}$ can vary appreciably based on the relative contributions to $\varepsilon$ from the formation and glide of sub-critical, thermally-nucleated (glissile) and pre-existing dislocations. The data in Table 1 demonstrates how a difference of two orders of magnitude in the pre-existing dislocation density suffices to produce a large difference in $U_{a p p}$ when all other parameters are held fixed. This was the rationale behind keeping the Burgers vector fixed and equal to that of ordinary dislocations in both cases in Table 1. It was done to ensure that changes in too many variables did not mask the underlying reason for the shift in $U_{a p p}$ and BDTT. In reality, many variables such as $b, \mu_{0}, c, U_{m}, M$ and $n_{0}$ are expected to be different for ordinary and superlattice dislocations in TiAl. The fact that the BDTT lies in the same range for both of them suggests that $U_{m}$ does not vary much in the two cases. The most significant difference between the two is the magnitude of the Burgers vector. According to the model, the apparent formation energy, $U_{n}$, scales with $b^{2}$. Thus, $U_{n}$ and $U_{a p p}$ are expected to be much larger for superlattice dislocations compared with the case of ordinary dislocations in agreement with observations.
The cooperative dislocation generation model also helps to explain why a high value of $U_{a p p}$ is not always associated with correspondingly high values of the BDTT. $U_{a p p}$ represents a composite value determined by the nucleation and glide of glissile dislocations below the BDTT. If a material contains a high density of pre-existing dislocations, a small density of thermally nucleated dislocations at temperatures below the BDTT may be sufficient for the onset of the cooperative instability. Such a process is feasible even if the apparent formation energy for thermal nucleation of glissile dislocations is somewhat larger than that for dislocation motion. This line of reasoning is supported by the data of [4] where it was found that the fracture toughness for superlattice dislocations was actually higher than that for ordinary dislocations but the variation of the toughness with temperature was similar to that for ordinary dislocations in the entire brittle regime. Such behavior is expected if the pre-existing density of superlattice dislocations is higher than that of ordinary dislocations but the activation energies for dislocation motion are similar in both cases.

In conclusion, a cooperative mechanism of dislocation generation above a critical temperature can give rise to massive dislocation activity of the type associated with the BDT. The strain-rate dependence of the critical temperature arises from the motion of pre-existing dislocations and dislocations which are 'thermally nucleated' below the critical temperature by the cooperative process. Depending on their contributions, the apparent activation energy associated with the brittleto-ductile transition temperature is either equal to or larger than the activation energy for dislocation motion.

\section{Acknowledgements}

This research was supported by the US Air Force Office of Scientific Research grant F49620-98-1-0245.

\section{References}

[1] J.F. Knott, Fundamentals of Fracture Mechanics, Butterworths, London, 1979.

[2] C. St. John, Philos. Mag. 32 (1975) 1193.

[3] S.G. Roberts, A.S. Booth, P.B. Hirsch, Mater. Sci. Eng. A176 (1994) 91.

[4] A.S. Booth, S.G. Roberts, Acta Mater. 45 (1997) 1045.

[5] M. Khantha, D.P. Pope, V. Vitek, Phys. Rev. Lett. 73 (1994) 684.

[6] M. Khantha, V. Vitek, Acta Mater. 45 (1997) 4675.

[7] M. Khantha, V. Vitek, Mater. Sci. Eng. A234-236 (1997) 629.

[8] M. Khantha, M. Ling, V. Vitek, Mater. Sci. Res. 5 (1999) 234.

[9] J. Cserti, Phys. Rev. B 60 (1999) 6175.

[10] J.M. Kosterlitz, D.J. Thouless, J. Phys. C Solid State Phys. 6 (1973) 1181. 
[11] A.P. Young, Phys. Rev. B 19 (1979) 1855.

[12] J.M. Kosterlitz, J. Phys. C Solid State Phys. 7 (1974) 1046.

[13] D.R. Nelson, B.I. Halperin, Phys. Rev. B 19 (1979) 2457.

[14] F. Lund, Phys. Rev. Lett. 69 (1992) 3084.

[15] M. Khantha, D.P. Pope, V. Vitek, Acta Mater. 45 (1997) 4687.
[16] M. Khantha, Scr. Metall. Mater. 31 (1994) 1355.

[17] M. Khantha, V. Vitek, Scr. Metall. Mater. 35 (1996) 285.

[18] V. Ambegoaker, B.I. Halperin, D.R. Nelson, E.D. Siggia, Phys. Rev. B 21 (1980) 1806. 